\title{
Mendelian randomization studies of brain MRl yield insights into the pathogenesis of neuropsychiatric disorders
}

\author{
Weichen Song ${ }^{1 \dagger}$, Wei Qian ${ }^{1 \dagger}$, Weidi Wang ${ }^{1,2}$, Shunying $\mathrm{Yu}^{1,2}$ and Guan Ning Lin ${ }^{1,2^{*}}$ (1) \\ From 19th International Conference on Bioinformatics 2020 (InCoB2020) \\ Virtual. 25-29 November 2020
}

\begin{abstract}
Background: Observational studies have identified various associations between neuroimaging alterations and neuropsychiatric disorders. However, whether such associations could truly reflect causal relations remains still unknown.

Results: Here, we leveraged genome-wide association studies (GWAS) summary statistics for (1) 11 psychiatric disorders (sample sizes varied from $n=9,725$ to 1,331,010); (2) 110 diffusion tensor imaging (DTI) measurement (sample size $n=17,706)$; (3) 101 region-of-interest (ROI) volumes, and investigate the causal relationship between brain structures and neuropsychiatric disorders by two-sample Mendelian randomization. Among all DTI-Disorder combinations, we observed a significant causal association between the superior longitudinal fasciculus (SLF) and the risk of Anorexia nervosa (AN) (Odds Ratio $[O R]=0.62,95 \%$ confidence interval: $0.50 \sim 0.76, P=6.4 \times 10^{-6}$ ). Similar significant associations were also observed between the body of the corpus callosum (fractional anisotropy) and Alzheimer's disease $\left(\mathrm{OR}=1.07,95 \% \mathrm{Cl}: 1.03 \sim 1.11, P=4.1 \times 10^{-5}\right)$. By combining all observations, we found that the overall $p$-value for DTI - Disorder associations was significantly elevated compared to the null distribution (Kolmogorov-Smirnov $P=0.009$, inflation factor $\lambda=1.37$ ), especially for DTI - Bipolar disorder (BP) $(\lambda=2.64)$ and DTI - AN $(\lambda=1.82)$. In contrast, for ROI-Disorder combinations, we only found a significant association between the brain region of pars triangularis and Schizophrenia $\left(\mathrm{OR}=0.48,95 \% \mathrm{Cl}: 0.34 \sim 0.69, P=5.9 \times 10^{-5}\right)$ and no overall $p$ value elevation for ROI-Disorder analysis compared to the null expectation.

Conclusions: As a whole, we show that SLF degeneration may be a risk factor for AN, while DTI variations could be causally related to some neuropsychiatric disorders, such as BP and AN. Also, the white matter structure might have a larger impact on neuropsychiatric disorders than subregion volumes.
\end{abstract}

Keywords: Neuroimaging, Neuropsychiatric disorders, Dysconnectivity, Anorexia nervosa, Superior longitudinal fasciculus

\footnotetext{
* Correspondence: nickgnlin@sjtu.edu.cn

'Weichen Song and Wei Qian contributed equally to the manuscript.

'Shanghai Mental Health Center, School of Biomedical Engineering, Shanghai

Jiao Tong University School of Medicine, Shanghai Jiao Tong University, 200030 Shanghai, China

${ }^{2}$ Shanghai Key Laboratory of Psychotic Disorders, 200030 Shanghai, China
}

(C) The Author(s). 2021 Open Access This article is licensed under a Creative Commons Attribution 4.0 International License, which permits use, sharing, adaptation, distribution and reproduction in any medium or format, as long as you give appropriate credit to the original author(s) and the source, provide a link to the Creative Commons licence, and indicate if changes were made. The images or other third party material in this article are included in the article's Creative Commons licence, unless indicated otherwise in a credit line to the material. If material is not included in the article's Creative Commons licence and your intended use is not permitted by statutory regulation or exceeds the permitted use, you will need to obtain permission directly from the copyright holder. To view a copy of this licence, visit http://creativecommons.org/licenses/by/4.0/. The Creative Commons Public Domain Dedication waiver (http://creativecommons.org/publicdomain/zero/1.0/) applies to the data made available in this article, unless otherwise stated in a credit line to the data. 


\section{Background}

Neuroimaging study is the most widely used procedure for studying brain disorders [1]. Many neuroimaging studies in the past quarter-century have revealed brain abnormalities in neuropsychiatric disorders [1, 2], which have served as the basis for biomarker discovery, clinical guidance, and investigations into the mechanisms of neuropsychiatric disorders [2, 3]. However, it is unclear whether such associations reflect disease causality [4]. One concern is that the spurious correlations [5] could emerge from indirect correlations with confounders such as medication, circadian and dietary changes, or falsepositive events. Another issue is the direction of causality: the neurotoxicity hypothesis [6] suggests that psychiatric illnesses have toxic effects on the central nervous system, which leads to structural alterations following disease onset [7]. This theory gained support from several observations in which neuroimaging abnormalities exhibited dynamic progression during the neuropsychiatric disorder course $[6,7]$. Under such theories, these associations should be utilized as clinical biomarkers rather than mechanism identifiers.

Several case-control studies with large sample sizes $[8,9]$ have found a significant correlation between neuroimaging alteration and neuropsychiatric disorder, yet still unable to distinguish the causality from the correlation. Longitudinal analyses have partially overcome the limitations of cross-sectional observational studies [10] for investigating the disease causality, such as detecting neuroimaging alterations in participants with the so-called high-risk status along with their progression $[11,12]$. However, these studies are still limited by our current definition of high-risk cohorts. For example, the onset of neuropsychiatric disorders may be far earlier than a clinically recognizable high-risk status, such that the associated abnormalities may include changes that occurred after the primary pathology.

Despite the longitudinal analysis focusing on the highrisk status, an alternative method for addressing the challenge of causality is Mendelian Randomization (MR) [13]. MR has been used to derive the relationships between peripheral inflammatory markers and schizophrenia [14] and between physical activity and depression [15] with great successes. By selecting genetic locus that is strongly associated with exposure, so-called instruments, MR separates subjects into high and low lifetime exposure groups according to their genotypes on the instruments [16], then compares the prevalence of outcomes between the high and low exposure groups. Such grouping is considered unbiased as genotypes are randomly determined during meiosis. Furthermore, a twosample Mendelian Randomization (MR) estimates instrument-exposure and instrument-outcome relationships in different cohorts to infer the exposureoutcome relationship, without the need for information on individuals [16]. The explosive growth of genomewide association studies (GWAS) offers opportunities for applying MR to solve the debate of causality in different clinical medicine fields. Recently, two GWAS from UK-Biobank (UKBB) $[17,18]$ revealed the genetic basis of brain structural measurements, providing an opportunity to address clinical neuroimaging studies' causality. In the present study, with the summary-statistics GWAS data of Magnetic resonance imaging (MRI) $(N=$ 17,706 and 19,629) and twelve neuropsychiatric disorders $(N=9,725$ to $1,331,010)$, we implemented a twosample MR approach to detect the causal relationship between white matter (WM) structures (diffusion tensor imaging [DTI]), brain subregion volumes (region of interest $[\mathrm{ROI}])$, and neuropsychiatric disorders. An overview of the study design is illustrated in Fig. 1.

\section{Results \\ Causal associations between DTI and neuropsychiatric Disorder}

After data harmonization, 1253/1320 (110*12) DTIDisorder pairs and 973/1212 (101*12) ROI-Disorder pairs had at least one strong instrument (SNP with $P<$ $5 \times 10^{-8}$ in DTI GWAS [17], Additional file 1) and were analyzed by MR (Additional file 2 and 3). We started by evaluating the DTI-Disorder association. In the inverse variance-weighted (IVW) analysis (outer layer of Fig. 2a), which required at least two instruments, one DTIDisorder pair achieved study-wide significance (SW, $P<$ 0.05/1320): superior longitudinal fasciculus Axial Diffusivity (SLF.AxD) - the risk of Anorexia nervosa (AN) (Fig. 2b; Table 1, odds ratio $[\mathrm{OR}]=0.62,95 \%$ confidence interval $[\mathrm{CI}], 0.50$ to $0.76, P=6.4 \times 10^{-6}$ ). Another DTIDisorder pair, body of corpus callosum Fractional Anisotropy (BCC.FA) - Alzheimer disease (AD), was close to reaching $\mathrm{SW}$ significance (Fig. 1c and Table 1, OR= $1.07,95 \% \mathrm{CI}, 1.03$ to $1.11, P=4.1 \times 10^{-5}$ ). Both associations had relatively consistent results across instruments (Fig. 2b, c), in accordance with the fact that no outlier was detected (Additional file 2). Five other DTI-Disorder pairs also reached single-disease significance $(P<0.05 /$ 110) (Table 1). No significant result was found for the Wald ratio (second layer of Fig. 1a) applied to DTIDisorder pairs with only one instrument.

A basic assumption of MR is that MR genetic instruments should only impact the outcome via the exposure and not any other pathway (horizontal pleiotropy) [16]. The existence of heterogeneity, which is introduced by outlier instruments, can also bias the MR estimation [19]. By applying various sensitivity tests, we confirmed that our results were not impacted by horizontal 


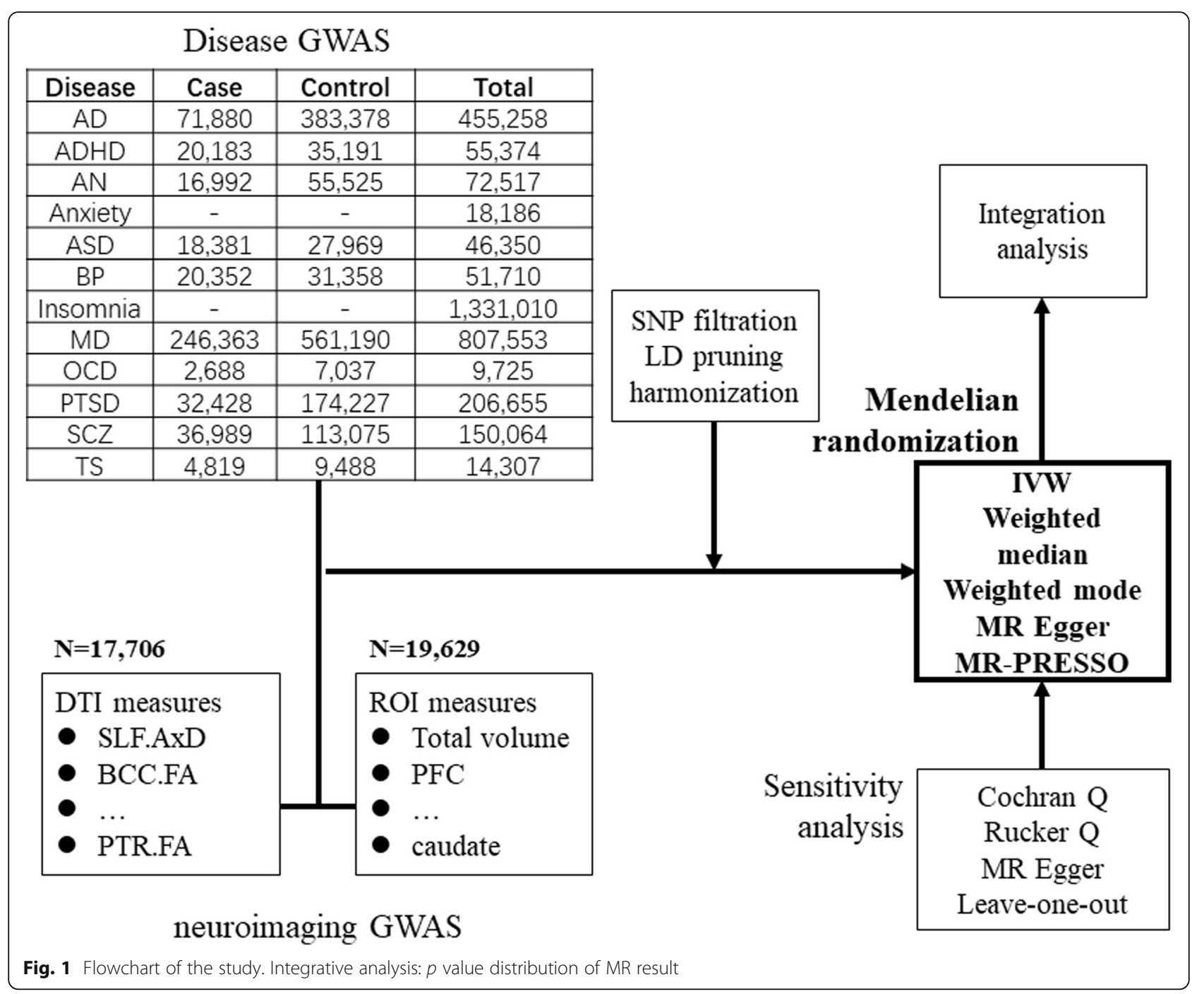

pleiotropy (Egger intercept $P>0.05$; the third layer of Fig. 1a and Additional file 2) or heterogeneity (modified Cochran's $Q$ test $P>0.05$; Table 1 ), or bidirectional effects (reverse MR $P>0.05$; Additional file 2). They also showed consistent trends across different MR methods, which are robust against pleiotropy and measurement error (see Method for detail) (except for Fornix.FA - bipolar disorder [BP]; Table 1). They also showed little directional pleiotropy, as indicated by funnel plots (Additional file 4). Neither MR-PRESSO nor leave-one-out tests found the impact of outliers on these DTI-Disorder pairs (Additional files 2 and 4). Taken together, these results confirmed the casual relation between SLF.AxD$\mathrm{AN}$, and suggested potential relations of other five DTIDisorder pairs.

Overall contribution of DTI on neuropsychiatric disorders Despite separate DTI-Disorder pairs that reached the significance threshold, we were also interested in whether DTI as a whole made a causal contribution to the diseases. To answer this question, we pooled the IVW $P$ value for all DTI-Disorder pairs and compared them to the null uniform distribution (Fig. 3a and Additional file 2). The distribution of IVW $P$ was significantly inflated (KS $P=0.009, \lambda=1.37$ ); this result persisted after removing heterogeneous DTIDisorder pairs (those with Cochran's $P<0.05$ ) (KS $P=0.002, \lambda=1.48$ ) or outlier instruments (SNPs with MR-PRESSO $P<0.05) \quad\left(\right.$ KS $P=8.0 \times 10^{-5}, \quad \lambda=1.49$ ). When we analyzed each disease separately ("Original" in Fig. 3b), BP (Fig. 3c) and AN showed the most significant inflation (Table 2). These results were also relatively stable against heterogeneity and outlier removal (Fig. 3b and Additional file 4). The permutation test confirmed that the results for BP (permutation $P$ value [pp] for KS test: 0.012 ; pp for $\lambda$ : 0.002 ) and AN (pp for $\lambda$ : 0.012) were not due to the bias inherent in the data or method. Additionally, 


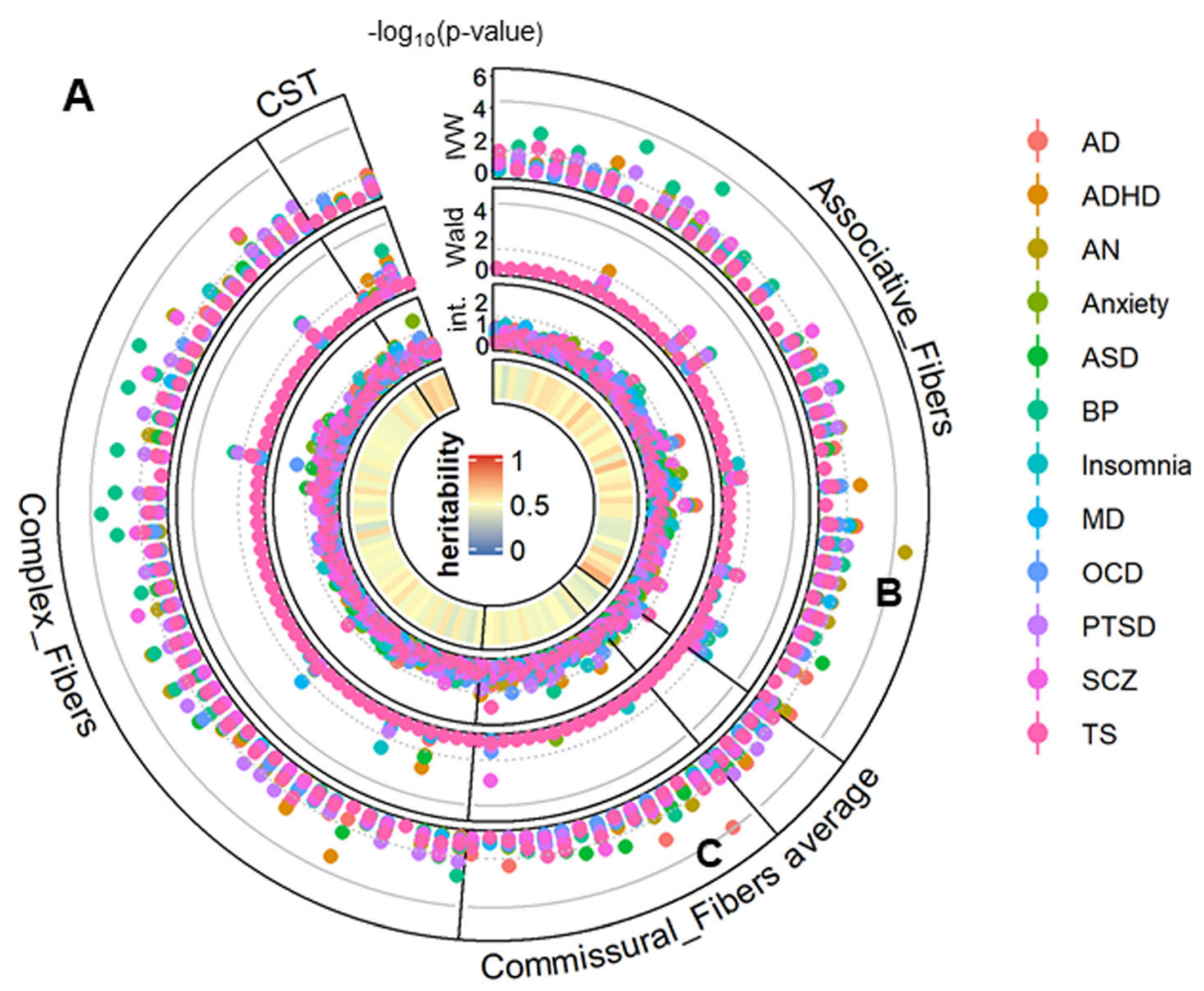

B

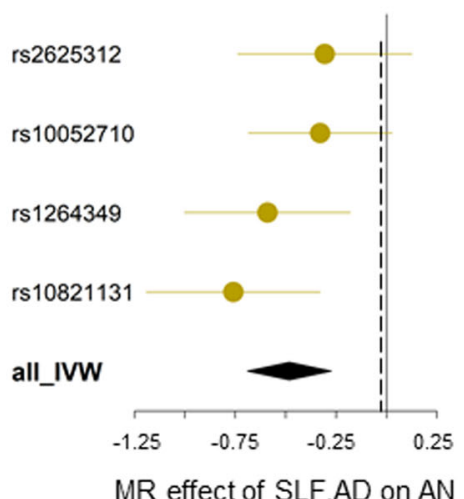

C

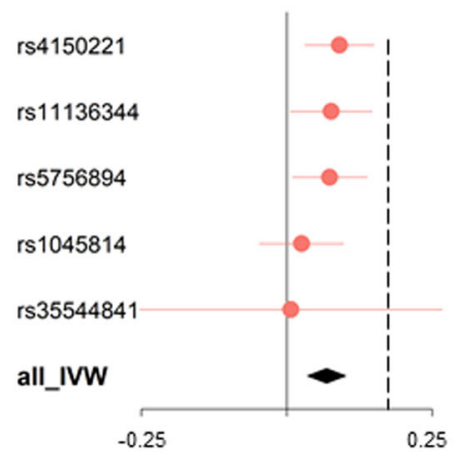

MR effect of BCC.FA on AD

Fig. 2 MR results for DTI-Disease associations. a: Each radius represents one DTI measures (110 in total: 22 white matter tracts $\times 5$ DTI parameters). From outer to inner $\operatorname{layer}:-\log _{10}(p)$ for MR-IWW; $-\log _{10}(p)$ for MR-Wald Ratio; - $\log _{10}(p)$ for intercepts (int.) of MR-Egger regression; heritability of each DTI measures. The dotted grey line indicated the nominal $p$ threshold (0.05); solid grey line indicated study-wide significance p threshold (0.05/1253). CST: Corticospinal tract. b\&c: Forest plots showing MR effect of Superior longitudinal fasciculus (axial diusivities) on Anorexia nervosa (SLF.AXD-AN) and body of corpus callosum (fractional anisotropy) on Alzheimer Disease (BDD.FA-AD). Each line showed the single SNP MR effects ( $95 \%$ confidence interval) estimated by Wald Ratio, and the last line showed the meta-analysis results calculated by IVW. Vertical dashed line indicated the Egger estimation of MR effect

heterogeneous DTI-Disorder pairs generally had nonsignificant MR results and did not contribute to inflation (Fig. 3d and Additional file 2). Thus, we concluded that DTI polymorphisms made an overall causal contribution to neuropsychiatric disorders, especially $\mathrm{BP}$ and $\mathrm{AN}$.

\section{Causal associations between brain volume and neuropsychiatric Disorders}

Similar to the DTI-Disorder analysis, we also assessed the ROI-Disorder association. 973/1212 (101*12) RD pairs had at least one strong instrument (SNP with $P<$ $5 \times 10^{-8}$ ) and were analyzed by MR. In the IVW analysis, 
Table 1 All DTI-Disorder pairs reaching Single Disease significant threshold ( $p<0.05$ after correction)

\begin{tabular}{lllllll}
\hline disease & DTI & IVW & Egger & Wtd.median & CochranQ & intercept \\
\hline AN & SLF.AxD & $-0.48\left(6.4 \times 10^{-6}\right)$ & $-0.02(0.97)$ & $-0.41(0.002)$ & $3.22(0.36)$ & $-0.04(0.52)$ \\
AD & BCC.FA & $0.07\left(4.1 \times 10^{-5}\right)$ & $0.18(0.31)$ & $0.08\left(3.6 \times 10^{-4}\right)$ & $2.20(0.70)$ & $-0.007(0.50)$ \\
BP & PTR.FA & $-0.31(0.0002)$ & $-0.22(0.53)$ & $-0.27(0.008)$ & $2.70(0.44)$ & $-0.010(0.76)$ \\
BP & FX.FA & $-0.31(0.0003)$ & $0.60(0.30)$ & $-0.27(0.01)$ & $6.60(0.47)$ & $-0.06(0.13)$ \\
BP & CGH.MD & $0.31(0.0003)$ & $0.11(0.70)$ & $0.29(0.004)$ & $3.13(0.37)$ & $0.02(0.48)$ \\
BP & RLIC.RD & $0.26(0.0004)$ & $0.11(0.67)$ & $0.22(0.01)$ & $6.92(0.33)$ & $0.01(0.55)$ \\
ADHD & ALIC.FA & $-0.44(0.0004)$ & $-1.33(0.41)$ & $-0.48(0.001)$ & $2.62(0.27)$ & $0.08(0.53)$ \\
\hline
\end{tabular}

Results were shown as estimate ( $P$ value). IVW Inverse Variance Weighted sum of Wald Ratio, Wtd.median MR effects estimated by weighted median, Intercept intercept of egger regression, intercept Egger intercept. The definition of DTI acronym are provided in the Method section

we found no SW significant results (outer layer of Fig. 3a and Additional file 3). The Wald ratio revealed a marginal SW result for pars triangularis (PT)-SCZ (OR = $0.48, P=5.93 \times 10^{-5}$ ) (second layer of Fig. 4a), which was driven by a single SNP (rs2279829). The only trait associated with rs2279829 in PhenoScanner [20] was daytime dozing or sleeping $\left(P=2.02 \times 10^{-6}\right)$. The SMRHEIDI test detected a significant MR effect in the same direction $\left(\mathrm{OR}=0.48, P=7.57 \times 10^{-4}\right)$ with no evidence of colocalization pleiotropy (HEIDI $P=0.84$ ). We estimated that per 1-SD increment in normalized PT volume, the risk of SCZ decreased by $52 \%(\mathrm{OR}=0.48)$. In conclusion, these results suggested a potential causal relationship between PT and SCZ. However, the validation of this relationship requires more work on potential pleiotropy.

The overall distribution of the IVW p-value did not significantly differ from the null distribution (Fig. 4b), even after removing heterogeneity (KS $P=0.12)$ and outliers (KS $P=0.07$ ). However, IVW p-value for SCZ showed significant inflation $(\lambda=1.90$, KS $P=0.001)$, and the result was significantly impacted by heterogeneity (Fig. 4c). Additionally, $10 \%(6 / 58)$ of the nominally significant RD pairs showed heterogeneity, and 3 contained outlier SNPs (Additional files 3 and 4). In conclusion, there was no evidence that ROI polymorphisms had a universal contribution to neuropsychiatric disorders.

\section{Discussion}

The issue of causality has long beleaguered clinical neuroimaging studies [4]. Confirmation of a causal change can provide insights into disease mechanisms at the circuit and region levels and may reveal useful biomarkers for predicting prognosis. Many current neuroimaging studies cannot directly draw this conclusion $[1,4]$, which partly limits the translation of their findings to clinical practice. In this study, we conducted hypothesis-free, data-driven MR analyses to assess the causal relationship between neuroimaging polymorphisms and neuropsychiatric disorders in an unbiased manner.
Our results showed that, in general, WM connectivity was more closely associated with the risk of neuropsychiatric disorders than gray matter volume (GMV) (Fig. 3a and 4b). Among neuropsychiatric disorders, BP showed the most significant association with genetically determined connectivity polymorphisms (Fig. 3b,c). These results support the dysconnectivity theory [21] of psychiatry, positing that major psychiatric disorders such as SCZ and BP have common WM abnormalities in their pathology [22]. According to this hypothesis, anatomic and neurodevelopmental changes that arise from neurotoxicity [6] are a consequence rather than a cause of the illness. Indirect evidence from a functional study of neuromodulation and myelination [21] and a casecontrol study of the high-risk state [12] supports the dysconnectivity theory. Research interest has now shifted from the region-of-origin to a connectome's concept [22], in which connections between brain regions rather than the regions themselves cause BP and other mental illnesses. Our finding that DTI is more closely associated with the onset of neuropsychiatric disorders than ROI provides supportive evidence for this paradigm shift.

Our study's top MR result was a novel risk factor for AN-namely, decreased SLF.AxD. A few studies have reported a decreased SLF integrity in AN patients [23, 24], but the results were inconsistent with the marginal effect size. As neuroimaging findings in AN patients are influenced by dietary and metabolic alterations [25], a causal change may not be manifested as a visible signal. Nonetheless, the SLF may contribute to body image distortion in the pathology of AN [24], probably through its connection to areas responsible for body image perception (prefrontal and parietal networks) and self-perception (inferior parietal lobe) [26]. Although abnormalities in both GMV [26] and WM connectivity [23, 24] have been observed, our results suggest that the latter is a primary cause. In contrast, the former is a consequence of neuromodulator mechanisms such as activity-dependent pruning [21].

The roles of $\mathrm{BCC}$ in $\mathrm{AD}$ and $\mathrm{PT}$ in $\mathrm{SCZ}-2$ marginally significant results from our MR analysis-have received 


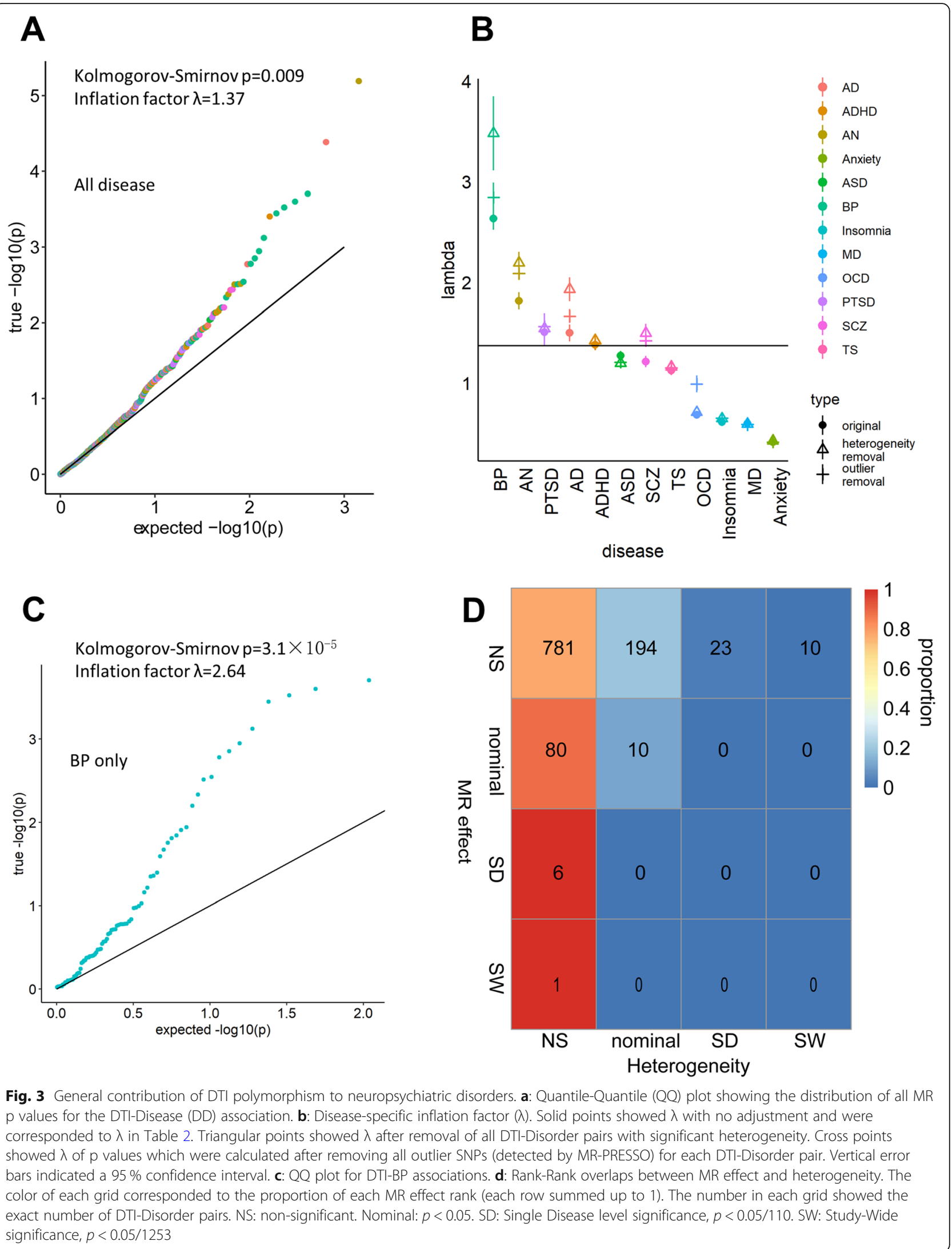


Table 2 Disease-specific $p$ value distribution

\begin{tabular}{llllll}
\hline disease & \multicolumn{2}{l}{ Subregion volume (ROI) } & & \multicolumn{2}{l}{ White matter structure (DTI) } \\
\cline { 2 - 3 } \cline { 5 - 6 } & lambda & p_ks & & lambda & p_ks \\
\hline AD & $1.15(1.07 \sim 1.23)$ & 0.92 & & $1.50(1.42 \sim 1.58)$ & 0.57 \\
ADHD & $1.79(1.74 \sim 1.86)$ & 0.21 & & $1.38(1.35 \sim 1.41)$ & 0.13 \\
AN & $1.20(1.12 \sim 1.30)$ & 0.72 & & $1.82(1.73 \sim 1.90)$ & 0.14 \\
anxiety & $1.04(1.01 \sim 1.09)$ & 0.35 & & $0.41(0.40 \sim 0.43)$ & 0.0090 \\
ASD & $1.08(1.05 \sim 1.11)$ & 0.36 & & $1.27(1.23 \sim 1.32)$ & 0.021 \\
BP & $1.10(1.07 \sim 1.15)$ & 0.49 & & $2.64(2.52 \sim 2.75)$ & $3.1 \times 10^{-5}$ \\
Insomnia & $1.42(1.38 \sim 1.47)$ & 0.29 & & $0.61(0.59 \sim 0.63)$ & 0.061 \\
MD & $1.10(1.05 \sim 1.16)$ & 0.49 & & $0.60(0.58 \sim 0.62)$ & $6.6 \times 10^{-4}$ \\
OCD & $0.95(0.91 \sim 0.99)$ & 0.09 & & $0.68(0.67 \sim 0.70)$ & 0.15 \\
PTSD & $0.81(0.75 \sim 0.89)$ & 0.49 & & $1.51(1.38 \sim 1.63)$ & $7.8 \times 10^{-8}$ \\
SCZ & $1.94(1.90 \sim 2)$ & 0.0011 & & $1.21(1.16 \sim 1.27)$ & 0.0075 \\
TS & $1.02(1.00 \sim 1.05)$ & 0.78 & & $1.12(1.08 \sim 1.17)$ & $9.3 \times 10^{-4}$ \\
\hline
\end{tabular}

Inflation factor (lambda) shown as estimate (95\% confidence interval). p_ks: $p$ value for Komogorov-Smirov test for uniform distribution

more attention in the literature than SLF in AN. However, both MR results should be interpreted with caution. BCC atrophy is widely observed in AD patients even at an early stage and reflects Wallerian degeneration and myelin breakdown [27]. However, our MR analysis revealed a reverse association: the FA of $\mathrm{BCC}$ was positively associated with $\mathrm{AD}$ risk $(\beta=0.07)$. One possible explanation for this discrepancy is that an enlarged $\mathrm{BCC}$ in early life is a risk factor for AD development at an older age, causing BCC atrophy after disease onset. Confirmation of such a complex theory requires more robust evidence from large-scale longitudinal studies. As for the PT-SCZ association, although it was validated by several additional analyses such as SMR-HEIDI and reverse MR, a single SNP-driven MR result is by nature suspect due to the unexplored pleiotropy $[16,28]$. Because the volume reduction of PT has been demonstrated in high-risk psychosis and first-episode SCZ patients [29], we suggest that the inferred causality between PT and SCZ is plausible.

There were some limitations to this study. Firstly, classic MR methods largely depend on high heritability and strong exposure instruments $[16,28]$. However, both heritability and number of instruments [17, 18] vary across the tested neuroimaging parameters, such that the power of MR is inconsistent across all DTI-Disorder and RD pairs. In fact, 67 DTI-Disorder and 239 RD pairs were discarded at the beginning of our analysis due to the absence of instruments. Even if there were causal links among them, they would not have been detected in our study. Thus, negative results for DD/RD pairs with limited instruments are not as convincing as positive results for those with adequate instruments. For the positive results, it should also be noticed that only the genetically regulated proportion of polymorphisms are associated with the disorders. Secondly, the original GWAS sample sizes may have impacted the MR results since estimation accuracy (i.e., standard error for effect size) for the instrument-outcome relationship is directly linked to the confidence interval of the MR effect estimate $[16,28]$. Since GWAS OCD and TS recruited fewer than 10,000 cases, their MR results were underpowered. In fact, several DD/RD pairs for OCD and TS had a large MR effect, but their wide CI range resulted in non-significant $P$ values. Future GWAS with a larger sample size, both for neuroimaging polymorphism and neuropsychiatric disorders, will provide a better chance to improve our understanding in this field.

\section{Conclusions}

In conclusion, our analysis results demonstrate that, in general, WM structures make a more significant contribution to the etiology of neuropsychiatric disorders-especially $\mathrm{BP}$ and $\mathrm{AN}$-than brain subregion volumes. SLF.AxD was causally related to AN; marginally significant relationships were also found between BCC.FA and $\mathrm{AD}$ and between PT and SCZ.

\section{Methods}

We obtained publicly available GWAS summary statistics for DTI, ROI, and neuropsychiatric without collecting any individual information. Ethics approval was obtained in each of the original studies; therefore, no further ethics approval was needed for the current study.

\section{Data collection and preprocessing}

The genetic instruments for DTI measurements have been previously described [17]. Briefly, the ENIGMADTI pipeline [30] was used to analyze UKBB diffusion MRI data for 17,706 European participants and generate 110 DTI parameters-namely, fractional anisotropy (FA), axial diffusivity (AxD), mean diffusivity, mode of anisotropy, and radial diffusivity, of $21 \mathrm{WM}$ tracts as well as their mean values. The genetic instrument for ROI volumes was also obtained from UKBB GWAS [18], which included 19,629 European participants and used the standard OASIS-30 Atropos template for registration and Mindboggle-101 atlas for labeling [31].

We collected GWAS summary statistics from the following neuropsychiatric studies on European cohorts: (1) Alzheimer disease (AD) [32]; (2) Attention-deficit/ hyperactivity disorder (ADHD) [33]; (3) Anorexia Nervosa (AN) [34]; (4) anxiety disorders (Categorical phenotype) [35]; (5) autism spectrum disorder (ASD) [36]; 6)bipolar disorder (BP) [37]; 7) insomnia [38] 8) major depression disorders (MD) [39]; 9) obsessive-compulsory disorder (OCD) [40]; 10) posttraumatic stress disorder (PTSD) [41]; 11) schizophrenia (SCZ) [42]; 12) Tourette 

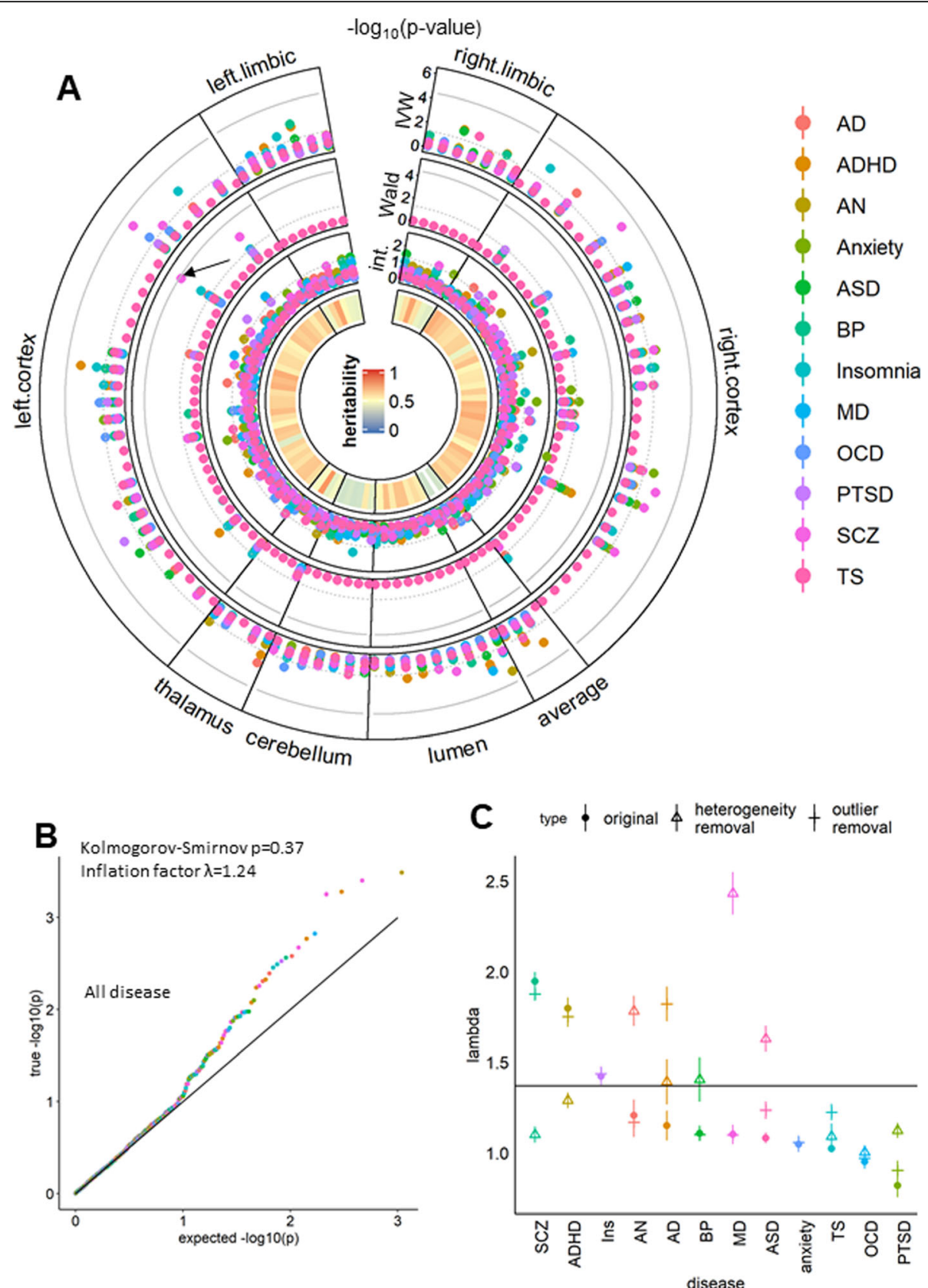

Fig. 4 MR results for ROI-Disease associations. a: Circos plot for ROI-Disease (RD) relations, similar to Fig. 1a. Arrow indicated result for pars triangularis-schizophrenia association, which was described in detail in the main text. b: QQ plot for all IVW p values of RD pairs, similar to Fig. 2a. c: Disease-specific inflation factor $(\lambda)$, similar to Fig. $2 b$

disorder (TD) [43] (Fig. 1). For GWAS from Psychiatric Genetic Consortium (PGC), there were few samples from UKB. For AD GWAS, the UKBB participants were not included in the case-control analysis. For other GWAS, we could not quantify the extent of sample overlap due to the lack of individual information. Since all GWAS used in the current study were conducted in European ancestry, we did not further adjust for the impact of population stratification.
For each DTI and ROI measurement, we retained single nucleotide polymorphisms (SNPs) with $P<5 \times 10^{-8}$ as strong instruments for MR; measurements without a strong instrument were discarded. We removed SNPs with linkage disequilibrium (LD) $\mathrm{r} 2 \geq 0.001$ for each measurement using reference LD data from the 1000 Genomes Project [44]. Data harmonization was applied independently for each DTI-disease (DD) and ROIdisease (RD) pair with the TwoSampleMR R package 
[45]. Since many of the GWAS summary statistics analyzed in this study did not provide allele frequency information, we did not exclude SNPs based on ambiguous strand error. For all binary phenotypes, we logtransformed the odds ratio to generate the $\beta$ value.

\section{Power calculation}

We calculated the variance in phenotype explained by each instrument by

$$
R^{2}=\frac{2 * E A F *(1-E A F) * \beta^{2}}{2 * E A F *(1-E A F) * \beta^{2}+2 * E A F *(1-E A F) * N * \operatorname{se}(\beta)^{2}}
$$

Where EAF was the effect allele frequency, $\beta$ was the effect size, $N$ was the sample size, and $\operatorname{se}(\beta)$ was the standard error of effect size. The F statistic was then denoted as

$$
F=\frac{R^{2} *(N-2)}{1-R^{2}}
$$

$R^{2}$ and $F$ were used to evaluate power for each instrument. For each RD and DTI-Disorder pair, we calculated the overall MR power using $\mathrm{mRnd}$ tool [46], assuming $\mathrm{OR}=1.3$ and type $\mathrm{I}$ error $=0.05$. The assumption of OR was based on the actual MR effects passing the significance threshold. We took this assumption because there is limited observational estimation of OR that is currently available. MR power and the number of valid instruments for each pair are recorded in Additional files 2 and 3. Details for all instruments are shown in Additional file 1.

\section{Calculation of MR effects}

For DD/RD pairs with at least two instruments, we performed a meta-analysis of each instrument's MR effect using the inverse variance-weighted (IVW) method. The results were considered preliminary results and were used for downstream analyses. For the top IVW findings, we additionally applied weighted mode [47], weighted median [13], and MR-Egger regression [48] approaches-which are relatively robust against horizontal pleiotropy [15] - to further confirm the validity of the MR effect. For DD/RD pairs with only one instrument, estimates based on the Wald ratio were considered preliminary results. For the top Wald ratio finding, we used summary data-based MR (SMR) [49] to confirm the existence of the MR effect and heterogeneity in dependent instruments (HEIDI) [49] to rule out the probability that the MR effect was driven by colocalization of the instrument with the effective locus.

Since both IVW and Wald ratio results were taken into account, the $p$-value was adjusted by the Bonferroni method by numbers of all DTI-Disorder pairs (1320) or ROI-Disorder pairs (1212).

\section{Sensitivity analysis}

The intercept of the Egger regression was used as an indicator of potential horizontal pleiotropy, while modified Cochran's Q for IVW and Rucker's Q for Egger regression [50] were used as an indicator of heterogeneity. We used MR pleiotropy residual sum and outlier (MR-PRES $\mathrm{SO}$ ) [19] with the number of permutations $=2500$ to detect potential outlier SNPs for each DD/RD pair and generate an overall $p$-value for heterogeneity; those with outlier(s) were reanalyzed by IVW after removing the outlier(s). We also applied leave-one-out tests for all top findings to further evaluate the effects of unknown outliers.

For all MR results, we tested the reverse MR effect (i.e., neuropsychiatric disorders as exposure and DTI/ ROI as outcome) by selecting SNPs with $P<5 \times 10^{-8}$ for each neuropsychiatric disorder as instruments. The absence of a reverse MR effect (IVW $P>0.05$ ) was considered as evidence for the validity of directionality.

\section{Analysis of the general causal contribution}

To assess the general contribution of DTI (ROI) polymorphisms to neuropsychiatric disorder, we pooled the IVW P values for all DTI-Disorder (RD) pairs and compared them to the null uniform distribution using quantile-quantile plots. A positive bias (inflation) from uniform distribution was considered as evidence for a general contribution. The significance of inflation was evaluated with the Kolmogorov-Smirnov (KS) test, while the extent of inflation was assessed with the inflation factor $\lambda$, which was calculated by chi-square regression using the GenABEL R package [51]. Since the inflation factor might be overestimated due to the small number of $P$ values, we shuffled SNP labels for BP and AN 1,000 times to carry out a permutation test. Permutation $P<$ 0.05 was considered evidence for significant inflation. These tests were also separately applied to each disease and repeated after removing heterogeneous results (those with Cochran's $P<0.05$ ) or outlier SNPs (those with MR-PRESSO $P<0.05)$.

\section{Abbreviations \\ 2SMR: 2-sample Mendelian randomization.; AD: Alzheimer Disease; AN: Anorexia Nervosa; BP: Bipolar disorder; DTI: Diffusion tensor imaging; ROI: Region-of-interest}

\section{Supplementary Information}

The online version contains supplementary material available at https://doi. org/10.1186/s12864-021-07661-8.

Additional file 1. Summary statistics for all instrument variables after data harmonization.

Additional file 2. Full results for DTI-Disease association analysis. Additional file 3. Full results for ROI-Disease association analysis. 
Additional file 4. Funnel plots for top MR findings, leave-one-out results for top DTI-Disorder pairs, general contribution of DTI to neuropsychiatric disease after sensitivity test adjustment, Rank-Rank overlaps between MR effect and heterogeneity for ROI-Disease analysis.

\section{Acknowledgements}

Not applicable.

\section{About this supplement}

This article has been published as part of BMC Bioinformatics Volume 22 Supplement 3, 2021: 19th International Conference on Bioinformatics 2020 (InCoB2020): genomics. The full contents of the supplement are available online at https://bmcgenomics.biomedcentral.com/articles/supplements/ volume-22-supplement-3.

\section{Authors' contributions}

G.N.L designed and supervised the study. S.Y, W.W collected the data. W.Q and W.S preprocessed and analyzed the data. W.S, W.Q, W.W, and S.Y interpreted the data. W.S wrote the manuscript. All authors read and approved the manuscript.

\section{Funding}

The study was supported by National Natural Science Foundation of China (No: 81971292 and 81671328); Program for Professor of Special Appointment (Eastern Scholar) at Shanghai Institutions of Higher Learning (Grant No. 1610000043); Innovation Research Plan supported by Shanghai Municipal Education Commission (Grant No. ZXWF082101). The funding bodies had no role in the study and collection, analysis, and interpretation of data and in writing the manuscript. Publication was funded by National Natural Science Foundation of China (No: 81971292).

\section{Availability of data and materials}

Disease GWAS summary data were downloaded from https://www.med.unc. edu/pgc/download-results/. GWAS summary of neuroimaging data were downloaded from https://github.com/BIG-S2/GWAS.

\section{Declarations}

Ethics approval and consent to participate Not applicable.

\section{Consent for publication}

Not applicable.

\section{Competing interests}

The authors declare that they have no competing interests.

\section{Received: 25 April 2021 Accepted: 29 April 2021}

Pulished: 2 June 2021

\section{References}

1. Etkin A. A reckoning and research agenda for neuroimaging in psychiatry. Am J Psychiatry. 2019;176:507-11.

2. Lui S, Zhou XJ, Sweeney JA, Gong Q. Psychoradiology: The frontier of neuroimaging in psychiatry. Radiology. 2016;281:357-72.

3. Aydin O, Unal Aydin P, Arslan A. Development of Neuroimaging-Based Biomarkers in Psychiatry. Adv Exp Med Biol. 2019:1192:159-95.

4. Etkin A. Addressing the causality gap in human psychiatric neuroscience. JAMA Psychiatry. 2018;75:3-4

5. Haig BD. What Is a Spurious Correlation? Underst Stat. 2003;2:125-32.

6. Weinberger DR, McClure RK. Neurotoxicity, neuroplasticity, and magnetic resonance imaging morphometry: What is happening in the schizophrenic brain? Arch Gen Psychiatry. 2002;59:553-8.

7. DeLisi LE. Defining the course of brain structural change and plasticity in schizophrenia. Psychiatry Res. 1999;92:1-9.

8. Hoogman M, Bralten J, Hibar DP, Mennes M, Zwiers MP, Schweren LSJ, et al. Subcortical brain volume differences in participants with attention deficit hyperactivity disorder in children and adults: a cross-sectional megaanalysis. The Lancet Psychiatry. 2017;4:310-9.
9. van Erp TGM, Walton E, Hibar DP, Schmaal L, Jiang W, Glahn DC, et al. Cortical Brain Abnormalities in 4474 Individuals With Schizophrenia and 5098 Control Subjects via the Enhancing Neuro Imaging Genetics Through Meta Analysis (ENIGMA) Consortium. Biol Psychiatry. 2018;84:644-54.

10. Pantelis C, Velakoulis D, McGorry PD, Wood SJ, Suckling J, Phillips L, et al. Neuroanatomical abnormalities before and after onset of psychosis: a crosssectional and longitudinal MRI comparison. Lancet. 2003;361:281-8.

11. Tang Y, Pasternak O, Kubicki M, Rathi Y, Zhang T, Wang J, et al. Altered cellular white matter but not extracellular free water on diffusion MRI in individuals at clinical high risk for psychosis. Am J Psychiatry. 2019; 176:820-8.

12. Roberts G, Perry A, Lord A, Frankland A, Leung V, Holmes-Preston E, et al. Structural dysconnectivity of key cognitive and emotional hubs in young people at high genetic risk for bipolar disorder. Mol Psychiatry. 2018;23:41321.

13. Bowden J, Davey Smith G, Haycock PC, Burgess S. Consistent Estimation in Mendelian Randomization with Some Invalid Instruments Using a Weighted Median Estimator. Genet Epidemiol. 2016;40:304-14.

14. Hartwig FP, Borges MC, Horta BL, Bowden J, Davey Smith G. Inflammatory Biomarkers and Risk of Schizophrenia. JAMA Psychiatry. 2017;74:1226.

15. Choi KW, Chen CY, Stein MB, Klimentidis YC, Wang MJ, Koenen KC, et al. Assessment of Bidirectional Relationships between Physical Activity and Depression among Adults: A 2-Sample Mendelian Randomization Study. JAMA Psychiatry. 2019;76:399-408.

16. Hemani G, Bowden J, Davey Smith G. Evaluating the potential role of pleiotropy in Mendelian randomization studies. Hum Mol Genet. 2018;27: R195-208.

17. Zhao B, Zhang J, Ibrahim JG, Luo T, Santelli RC, Li Y, et al. Large-scale GWAS reveals genetic architecture of brain white matter microstructure and genetic overlap with cognitive and mental health traits $(n=17,706)$. Mol Psychiatry. 2019. https://doi.org/10.1038/s41380-019-0569-z.

18. Zhao B, Luo T, Li T, Li Y, Zhang J, Shan Y, et al. Genome-wide association analysis of 19,629 individuals identifies variants influencing regional brain volumes and refines their genetic co-architecture with cognitive and mental health traits. Nat Genet. 2019;51:1637-44.

19. Verbanck $M$, Chen $C Y$, Neale B, Do R. Detection of widespread horizontal pleiotropy in causal relationships inferred from Mendelian randomization between complex traits and diseases. Nat Genet. 2018:50:693-8.

20. Staley JR, Blackshaw J, Kamat MA, Ellis S, Surendran P, Sun BB, et al. PhenoScanner: a database of human genotype-phenotype associations. Bioinformatics. 2016;32:3207-9.

21. Friston K, Brown HR, Siemerkus J, Stephan KE. The dysconnection hypothesis (2016). Schizophr Res. 2016;176:83-94.

22. Perry A, Roberts G, Mitchell PB, Breakspear M. Connectomics of bipolar disorder: a critical review, and evidence for dynamic instabilities within interoceptive networks. Mol Psychiatry. 2019;24:1296-318.

23. Phillipou A, Carruthers SP, Di Biase MA, Zalesky A, Abel LA, Castle DJ, et al. White matter microstructure in anorexia nervosa. Hum Brain Mapp. 2018;39: 4385-92.

24. Barona M, Brown M, Clark C, Frangou S, White T, Micali N. White matter alterations in anorexia nervosa: Evidence from a voxel-based meta-analysis. Neurosci Biobehav Rev. 2019;100:285-95.

25. Treasure J, Zipfel S, Micali N, Wade T, Stice E, Claudino A, et al. Anorexia nervosa. Nat Rev Dis Prim. 2015;1:1-21.

26. Gaudio S, Quattrocchi CC. Neural basis of a multidimensional model of body image distortion in anorexia nervosa. Neurosci Biobehav Rev. 2012;36: 1839-47.

27. Di Paola M, Spalletta G, Caltagirone C. In vivo structural neuroanatomy of corpus callosum in Alzheimer's disease and mild cognitive impairment using different MRI techniques: A review. J Alzheimer's Dis. 2010;20:67-95.

28. Burgess S, Bowden J, Fall T, Ingelsson E, Thompson SG. Sensitivity analyses for robust causal inference from mendelian randomization analyses with multiple genetic variants. Epidemiology. 2017;28:30-42.

29. Iwashiro N, Suga M, Takano Y, Inoue H, Natsubori T, Satomura Y, et al. Localized gray matter volume reductions in the pars triangularis of the inferior frontal gyrus in individuals at clinical high-risk for psychosis and first episode for schizophrenia. Schizophr Res. 2012;137:124-31.

30. Jahanshad N, Kochunov PV, Sprooten E, Mandl RC, Nichols TE, Almasy L, et al. Multi-site genetic analysis of diffusion images and voxelwise heritability analysis: A pilot project of the ENIGMA-DTI working group. Neuroimage. 2013;81:455-69. 
31. Klein A, Ghosh SS, Bao FS, Giard J, Häme Y, Stavsky E, et al. Mindboggling morphometry of human brains. PLoS Comput Biol. 2017;13:e1005350.

32. Jansen IE, Savage JE, Watanabe K, Bryois J, Williams DM, Steinberg S, et al. Genome-wide meta-analysis identifies new loci and functional pathways influencing Alzheimer's disease risk. Nat Genet. 2019;51:404-13.

33. Demontis D, Walters RK, Martin J, Mattheisen M, Als TD, Agerbo E, et al. Discovery of the first genome-wide significant risk loci for attention deficit/ hyperactivity disorder. Nat Genet. 2019;51:63-75.

34. Watson HJ, Yilmaz Z, Thornton LM, Hübel C, Coleman JRI, Gaspar HA, et al. Genome-wide association study identifies eight risk loci and implicates metabo-psychiatric origins for anorexia nervosa. Nat Genet. 2019;51:1207-14.

35. Otowa T, Hek K, Lee M, Byrne EM, Mirza SS, Nivard MG, et al. Meta-analysis of genome-wide association studies of anxiety disorders. Mol Psychiatry. 2016;21:1391-9.

36. Grove J, Ripke S, Als TD, Mattheisen M, Walters RK, Won H, et al. Identification of common genetic risk variants for autism spectrum disorder. Nat Genet. 2019;51:431-44.

37. Stahl EA, Breen G, Forstner AJ, McQuillin A, Ripke S, Trubetskoy V, et al. Genome-wide association study identifies 30 loci associated with bipolar disorder. Nat Genet. 2019;51:793-803.

38. Jansen PR, Watanabe K, Stringer S, Skene N, Bryois J, Hammerschlag AR, et al. Genome-wide analysis of insomnia in 1,331,010 individuals identifies new risk loci and functional pathways. Nat Genet. 2019;51:394-403.

39. Howard DM, Adams MJ, Clarke TK, Hafferty JD, Gibson J, Shirali M, et al. Genome-wide meta-analysis of depression identifies 102 independent variants and highlights the importance of the prefrontal brain regions. Nat Neurosci. 2019;22:343-52.

40. Arnold PD, Askland KD, Barlassina C, Bellodi L, Bienvenu OJ, Black D, et al. Revealing the complex genetic architecture of obsessive-compulsive disorder using meta-analysis. Mol Psychiatry. 2018;23:1181-8.

41. Nievergelt CM, Maihofer AX, Klengel T, Atkinson EG, Chen C-Y, Choi KW, et al. International meta-analysis of PTSD genome-wide association studies identifies sex- and ancestry-specific genetic risk loci. Nat Commun. 2019;10: 4558.

42. Ripke S, Neale BM, Corvin A, Walters JTR, Farh KH, Holmans PA, et al. Biological insights from 108 schizophrenia-associated genetic loci. Nature. 2014:511:421-7.

43. Yu D, Sul JH, Tsetsos F, Nawaz MS, Huang AY, Zelaya I, et al. Interrogating the Genetic Determinants of Tourette's Syndrome and Other Tic Disorders Through Genome-Wide Association Studies. Am J Psychiatry. 2019;176:217-27.

44. 1000 Genomes Project Consortium. A global reference for human genetic variation. Nature. 2015;526:68-74.

45. Hemani $\mathrm{G}$, Zheng J, Elsworth B, Wade KH, Haberland V, Baird D, et al. The MR-base platform supports systematic causal inference across the human phenome. Elife. 2018;7:e34408.

46. Brion M-JA, Shakhbazov K, Visscher PM. Calculating statistical power in Mendelian randomization studies. Int J Epidemiol. 2013;42(5):1497-501.

47. Hartwig FP, Smith GD, Bowden J. Robust inference in summary data Mendelian randomization via the zero modal pleiotropy assumption. Int J Epidemiol. 2017:46:1985-98.

48. Bowden J, Davey Smith G, Burgess S. Mendelian randomization with invalid instruments: effect estimation and bias detection through Egger regression. Int J Epidemiol. 2015;44:512-25.

49. Wu Y, Zeng J, Zhang F, Zhu Z, Qi T, Zheng Z, et al. Integrative analysis of omics summary data reveals putative mechanisms underlying complex traits. Nat Commun. 2018;9:918.

50. Ucker GR"', Schwarzer G, Carpenter JR, Binder H, Schumacher M. Treatmenteffect estimates adjusted for small-study effects via a limit meta-analysis. Biostatistics. 2011;12:122-42.

51. Aulchenko YS, Ripke S, Isaacs A, van Duijn CM. GenABEL: An R library for genome-wide association analysis. Bioinformatics. 2007;23:1294-6.

\section{Publisher's Note}

Springer Nature remains neutral with regard to jurisdictional claims in published maps and institutional affiliations.

\section{Ready to submit your research? Choose BMC and benefit from:}

- fast, convenient online submission

- thorough peer review by experienced researchers in your field

- rapid publication on acceptance

- support for research data, including large and complex data types

- gold Open Access which fosters wider collaboration and increased citations

- maximum visibility for your research: over $100 \mathrm{M}$ website views per year

At BMC, research is always in progress.

Learn more biomedcentral.com/submissions 